Archives de sciences sociales des religions

176 | octobre-décembre 2016

Bulletin Bibliographique

\title{
Église catholique et Russie en révolution
}

Étienne Fouilloux

\section{(2) OpenEdition}

Journals

Édition électronique

URL : http://journals.openedition.org/assr/28141

DOI : $10.4000 /$ assr. 28141

ISSN : $1777-5825$

Éditeur

Éditions de l'EHESS

Édition imprimée

Date de publication : 31 décembre 2016

Pagination : 169-176

ISSN : 0335-5985

\section{Référence électronique}

Étienne Fouilloux, «Église catholique et Russie en révolution », Archives de sciences sociales des religions [En ligne], 176 | octobre-décembre 2016, mis en ligne le 01 janvier 2019, consulté le 03 janvier 2020. URL : http://journals.openedition.org/assr/28141 ; DOI : 10.4000/assr.28141 


\title{
Étienne Fouilloux Église catholique et Russie en révolution
}

\author{
À propos de : \\ COeuRé Sophie, Pierre Pascal. La Russie entre christianisme et commu- \\ nisme, Lausanne, Les Éditions Noir sur Blanc, 2014, 416 p., index. \\ Pettinaroli Laura, La politique russe du Saint-Siège (1905-1939), \\ Rome, Publications de l'École française de Rome, coll. «Biblio- \\ thèque des Écoles françaises d'Athènes et de Rome ", 2015, 938 p., \\ index.
}

Entre 1905 et 1917, deux guerres et trois révolutions viennent à bout de l'empire pluriséculaire des Romanov. Elles accouchent d'un pouvoir inédit qui entend, par la contrainte plus que par la persuasion, instaurer à marches forcées le communisme en Russie. Le régime dirigé par Lénine, puis par Staline, est un régime totalitaire qui ne tolère aucune opposition, de quelque nature qu'elle soit, politique, sociale, culturelle ou religieuse. Alors que l'empire des tsars était intimement lié à l'Église orthodoxe, dont un concile a restauré la fonction patriarcale en 1917-1918 ${ }^{1}$, la dictature communiste lui inflige une persécution sans précédent qui la menace de disparition en 1939. Plutôt que par l'athéisme d'État officiellement prôné, elle remplace ce christianisme d'État par une religion de substitution, celle de l'homme nouveau à construire et de ses chefs qu'elle entoure d'un véritable culte. Longtemps sous-estimée des historiens, faute d'accès aux sources, l'histoire religieuse de la Russie soviétique est aujourd'hui assez bien connue, l'affrontement entre le pouvoir communiste et le patriarcat de Moscou notamment ${ }^{2}$.

L'Église catholique aurait pu estimer à bon droit que cet affrontement ne la concernait pas et n'accorder par voie de conséquence à la Russie qu'un intérêt limité. Elle n'a en effet pas été ménagée par le pouvoir tsariste au XIX siècle. Église latine à dominante polonaise ou lithuanienne, elle était considérée comme un corps étranger dans l'empire, surveillée de près et privée de liberté jusqu'aux réformes de 1905-1906. Après le traité de Brest-Litovsk qui entérine, en mars

1. Hyacinthe Destivelle, Le concile de Moscou (1917-1918). La création des institutions conciliaires de l'Église orthodoxe russe, Paris, Éditions du Cerf, 2006.

2. Voir par exemple, Adriano Roccucci, Stalin e il patriarca: Chiesa ortodossa et potere sovietico, 1917-1958, Turin, Einaudi, 2011.

ARCHIVES DE SCIENCES SOCIALES DES RELIGIONS 176 (octobre-décembre 2016), p. 169-175 
1918, la sécession de la Pologne et des Pays baltes, elle n’est plus dans la Russie soviétique qu'une minorité, faible numériquement et sans grande influence. Elle pâtit certes, elle aussi, de la politique antireligieuse du régime, mais sans y apparaître en première ligne, sauf lors du procès de 1923 intenté à quelques-uns de ses principaux dignitaires.

Or, c'est tout le contraire qui se produit : loin de décourager le monde catholique par sa politique antireligieuse, la jeune expérience soviétique y suscite un vif intérêt, et ce de deux manières différentes, qu'illustrent bien les deux ouvrages recensés. Quelques catholiques notoires, peu nombreux, mais de forte personnalité, s'enthousiasment pour une révolution radicale qui satisfait leur désir d'égalité et de fraternité : pour une durée plus ou moins longue, ils en deviennent, sur place ou en Occident, des compagnons de route. Le Saint-Siège, pour sa part, ne nourrit guère d'illusions de ce type ; mais on sait qu'il a pris son temps avant de condamner le communisme : l'encyclique de Pie XI Divini Redemptoris le dénonce comme "intrinsèquement pervers " en mars 1937, soit près de vingt ans après sa victoire à Petrograd et à Moscou. Entre temps, Rome a exploré diverses voies permettant de lever l'interdit qui pesait sur l'apostolat catholique dans l'empire des tsars et de négocier avec le pouvoir soviétique un statut, ou à défaut un modus vivendi, pour le catholicisme dans la Russie bolchevique. Il ne fait certes qu'appliquer à celle-ci la politique concordataire adoptée avec tous les États issus de la Première Guerre mondiale, mais ces pourparlers surprennent, vu l'hostilité déclarée de ses interlocuteurs envers la religion.

Pierre Pascal, né en 1890, est la figure la plus représentative des catholiques attirés par la révolution bolchevique. Ce jeune intellectuel d'origine bourgeoise, reçu à l'École normale supérieure en 1910 et agrégé de lettres en 1913, est promis à une brillante carrière universitaire. En quête d'un idéal plus exaltant que celui que lui offre son milieu, il s'est pris de passion pour les richesses spirituelles de la Russie, découvertes au cours de ses études dans la mouvance du lazariste Fernand Portal, apôtre de l'union des chrétiens séparés. C'est dans cet esprit qu'il part pour Petrograd en 1916, comme membre de la mission militaire détachée par la France auprès de l'allié russe. Le lieutenant Pascal y vit avec un enthousiasme croissant les soubresauts qui aboutissent à la chute du tsarisme, puis au coup d'État de Lénine et des siens. Aussi décide-t-il de rester en Russie quand les autorités françaises procèdent au retrait de la mission, et de mettre au service du nouveau régime ses compétences diplomatiques et linguistiques. Ce disciple de saint Thomas d'Aquin ne devient pas marxiste pour autant et ne renie pas sa foi catholique : son adhésion aux Soviets tient plus du sentiment que de la raison, de la ferveur que de la dialectique. Sans être un acteur de premier plan, il fréquente d'assez près les dirigeants communistes pour que son journal, édité après coup non sans avoir été remanié, soit une source précieuse sur l'évolution de la Russie soviétique dans les années $1920^{3}$.

3. Pierre Pascal, Mon journal de Russie. 1. À la mission militaire française, 1916-1918; 2. En communisme, 1918-1922 ; 3. Mon état d'âme, 1922-1926 ; 4. Russie 1927, Lausanne, L'Âge d'Homme, 1975-1982. 
Sophie Coeuré, qui a participé à l'édition (intégrale) du dernier volume ${ }^{4}$, y puise abondamment, comme dans bien d'autres fonds d'archives, pour reconstituer de manière très vivante les années moscovites de son héros et le milieu cosmopolite dans lequel il vit. Obligé de donner des gages pouvant aller jusqu'aux limites de la délation, il déplore pourtant la répression policière qui frappe les cercles oppositionnels dont il est proche : son ami Boris Souvarine, son beau-frère Victor Serge ou la famille de sa compagne Evguenia Roussakova. Jointes à une vie matérielle de plus en plus précaire, la faillite de son utopie révolutionnaire et les craintes pour sa liberté le conduisent à faire des démarches pour rentrer en France. De retour à Paris en 1933, il reprend ses travaux universitaires, qui aboutissent, en 1939, à la soutenance de sa grande thèse sur le schisme des vieux-croyants, et qui lui permettent d'intégrer l'enseignement supérieur, à Lille, puis à l'École des langues orientales, et enfin à la Sorbonne en 1950. Il devient ainsi l'un des meilleurs slavisants français, tout en maintenant un blackout presque complet sur son passé soviétique et en refusant tout engagement militant. Il faut attendre sa retraite, en 1960, et surtout l'ébranlement du communisme, pour que ce passé réapparaisse et que Pierre Pascal devienne en France, jusqu'à sa mort en 1983, l'un des " passeurs " privilégiés avec les dissidents d'un monde en voie de disparition. Sa vie étant un roman, du moins jusqu'à son retour à Paris, la biographie que lui consacre Sophie Coeuré, dotée de toutes les qualités universitaires requises, se lit comme un roman. Elle promeut Pierre Pascal au rang de grand témoin du chemin accidenté parcouru par la Russie au $\mathrm{XX}^{\mathrm{e}}$ siècle, de Nicolas II à Lénine et à Soljénitsyne.

L'ambition de Laura Pettinaroli est différente. Elle vise à éclairer, grâce aux archives des pontificats de Pie X (1903-1914), de Benoît XV (1914-1922) et surtout de Pie XI (1922-1939), les diverses facettes des rapports entre l'Église catholique et la Russie, tsariste puis communiste, entre les soubresauts révolutionnaires de 1905 et l'annexion par Staline, après le pacte Ribbentrop-Molotov d'août 1939, de vastes terres chrétiennes en Pologne et dans les Pays baltes. Issu d'une thèse de doctorat, ce travail monumental la dépasse de beaucoup, car il réussit le tour de force d'intégrer les nombreux acquis de la recherche depuis l'ouverture des archives vaticanes et soviétiques. Ses 100 pages de sources et de bibliographie, ainsi que ses 20 pages d'index, précieux instruments de travail, témoignent de l'ampleur de l'effort accompli : en six langues, français, anglais, allemand, italien, russe et polonais, Laura Pettinaroli a tout lu sur son sujet et tout assimilé ${ }^{5}$. Aussi livre-t-elle une histoire globale des relations entre Rome et la Russie dans le premier tiers du $\mathrm{XX}^{\mathrm{e}}$ siècle, selon trois approches complémentaires.

4. Pierre Pascal, Journal de Russie, 1928-1929, édité et annoté par Jacques Catteau, Sophie Coeuré et Julie Bouvard, Lausanne, Éditions Noir sur Blanc, 2014.

5. Il faut savoir gré aux publications de l'École française de Rome, dont Laura Pettinaroli a été l'élève, d'avoir édité un tel ouvrage (1,5 kilo sur la balance...), avec le luxe, de plus en plus rare malheureusement, des notes en bas de page. 
L'histoire qu'elle donne à lire est d'abord une histoire diplomatique de facture classique, fondée sur l'exploitation des fonds des chancelleries, mais qui révèle aussi le rôle décisif d'intermédiaires méconnus : Mgr Casimir Skirmunt par exemple, prélat polonais influent au début du siècle, ou le jésuite américain Edmund Walsh, pièce importante du réseau pontifical russe dans les années 1920. Mais le "politique» du titre ne doit pas être entendu dans un sens étroit. Une deuxième approche, de type institutionnel, s'attache à décrire le dispositif mis en place par l'Église catholique pour préparer le retour de la Russie orthodoxe dans son giron: dicastère dédié de façon spécifique à l'Orient chrétien (1917), Institut pontifical oriental (1917), séminaire russe Saint-Basile de Lille (1923), Commission pontificale pro Russia (1925), séminaire russe de Rome (1929), etc. Dans ce registre également, l'analyse fait toute sa place aux hommes qui ont suscité ou qui dirigent ces divers organismes. Au cœur du travail de Laura Pettinaroli se trouve ainsi le jésuite français Michel Bourguignon d'Herbigny dont elle éclaire de façon décisive l'ascension russe et la chute, des années 1920 aux années 1930. Une troisième approche, d'anthropologie religieuse, met en valeur les démarches intellectuelles et spirituelles qui sous-tendent cette passion catholique surprenante pour la Russie: Rome n'a pas seulement négocié avec Petrograd et Moscou, ni bâti un réseau d'institutions ad hoc, elle a aussi développé un discours théologique et des formes de prière à destination de la Russie. C'est de la combinaison, variable selon les périodes, entre ces trois approches que naît l'histoire globale de la relation dissymétrique que Laura Pettinaroli nous fait découvrir.

Son travail est distribué, d'une façon qui n'est guère contestable, en trois grandes phases chronologiques. Au cours de la première, qui va des soubresauts de 1905 à ceux de 1917, la dominante est diplomatique. Après avoir dressé le tableau de la situation, peu enviable, des catholiques dans l'Empire russe au début du XX $\mathrm{X}^{\mathrm{e}}$ siècle, Laura Pettinaroli décrit les efforts du Saint-Siège pour élargir la liberté religieuse consentie en 1906 aux dimensions d'un véritable statut pour le catholicisme. Efforts vains jusqu'à la chute du tsarisme, tant une réaction autoritaire suit de peu ses ouvertures libérales. Efforts qui paraissent couronnés de succès après mars 1917, avec le gouvernement provisoire, mais sans lendemains du fait de la révolution bolchevique. À une époque, le pontificat de Pie X, où les efforts de rapprochement interconfessionnel sont vite suspects d'hérésie moderniste, les réseaux russophiles demeurent inchoatifs et l'engouement pour la Russie ne dépasse pas quelques cercles limités, en France, en Belgique ou en Italie. C'est pourtant au cours de ces années d'avant-guerre que naissent les vocations russes d'un Pierre Pascal ou d'un Michel d'Herbigny.

La seconde phase, entre l'arrivée au pouvoir des bolcheviques et le raidissement antireligieux de Staline à la fin des années 1920, voit au contraire l'apogée de la passion catholique pour la Russie. Considérant le tsarisme et son lien privilégié à l'Église orthodoxe comme l'obstacle principal à la pénétration du 
catholicisme dans l'empire, le Saint-Siège n'interprète pas sa chute comme une catastrophe, bien au contraire. Certes, son successeur communiste n'est guère fréquentable, mais ne faut-il pas négocier avec le diable pour le bien de l'Église ? Le peu de succès des pourparlers entrepris n'empêche pas Rome de reconstituer de façon semi-clandestine la hiérarchie catholique en Russie ni de créer de toutes pièces un réseau d'institutions pour lui préparer des prêtres et des religieux. La conversion de la Russie et son retour dans le giron catholique occupent alors une place de choix dans un unionisme romain en plein essor. En tandem avec Pie XI, personnellement intéressé au sort de la Russie depuis son ambassade polonaise de 1918-1921, Mgr d'Herbigny est le principal protagoniste de cet effort pour donner corps à ce qui fait figure, après coup, de mirage russe.

Car le mirage se brise sur la réalité. Les dirigeants communistes ne sont pas disposés à accorder pleine liberté au catholicisme en Russie : la hiérarchie d'Herbigny est vite démantelée par la Guépéou. Le Saint-Siège, de son côté, hésite à reconnaître un régime issu de la subversion. D'autant que sa stalinisation s'accompagne d'une répression religieuse accrue, symbolisée par le décret sur les cultes de 1929. La persécution entraîne une protestation internationale dans laquelle le Saint-Siège, pour la première fois depuis 1917, prend une place remarquée, en mobilisant les fidèles dans une prière de réparation. Plus de négociations ni même de relations à caractère diplomatique, dans cette dernière phase. Quant au réseau des institutions pour la Russie, il entre en sommeil, à l'exemple de la Commission pro Russia, dont d'Herbigny est exclu en 1933. Le mirage d'une possible conversion catholique de la Russie s'estompant, l'heure est au combat, tout à la fois social et spirituel. Social ? Un anticommunisme catholique, dont l'encyclique de 1937 est l'acmé, se développe en prolongement de la doctrine sociale de l'Église. Spirituel ? Rome mobilise des dévotions traditionnelles, et la dévotion nouvelle à sainte Thérèse de Lisieux, dans une prière à caractère eschatologique pour la Russie, qui ne craint pas d'exalter le martyre des victimes de la persécution. Au moment où de gros bataillons polonais et lithuaniens viennent à leur corps défendant le rejoindre, le catholicisme est exsangue en Russie soviétique : la "politique russe » du Saint-Siège semble se solder par un échec.

Pour chacune des périodes, Laura Pettinaroli tisse adroitement les trois approches adoptées en une démonstration dont on ne peut qu'évoquer allusivement la richesse. Son travail considérable, qui allie de façon heureuse analyse de documents inédits et synthèse des recherches en cours, fera date. Et il suggère nombre de questions prouvant sa fécondité. On se contentera ici d'en évoquer deux. La première se situe à l'intérieur de la perspective adoptée : la «politique russe » du Saint-Siège. Mais quelle place occupe celle-ci dans ses préoccupations, dont l'extension contemporaine du réseau des nonciatures et délégations apostoliques montre qu'elles englobent désormais bien des pays dans tous les 
continents $^{6}$ ? Laura Pettinaroli est convaincante quand elle souligne l'opportunité offerte par l'ébranlement puis la chute du tsarisme, entre 1905 et 1917, ou le surgissement consécutif de la Russie dans la conscience catholique au cours des années 1920 : implication personnelle du pape Pie XI, création avec la Commission pro Russia d'un organisme spécifique, procédures extraordinaires sous la houlette de Mgr d'Herbigny, développement parmi les fidèles d'un attrait pour les fastes liturgiques et spirituels de la grande nation chrétienne en butte à un athéisme d'État. Mais cet attrait survit-il à l'échec de la "politique russe » et à la tentative d'éradication dont le catholicisme est victime dans la Russie de Staline ? On peut en douter, même si cette "politique " retrouve brièvement des couleurs pendant la Seconde Guerre mondiale, avant la seconde glaciation stalinienne. Bien des indices suggèrent que c'est la Chine de Mao qui prend à partir de 1950 la place de la Russie comme horizon messianique de conquête ou de conversion, mais aussi de persécution et de martyre suscitant nombre de vocations, comme la Russie antérieurement. Alors que la "politique » française, italienne ou allemande du Saint-Siège est une constante, sa "politique russe " ne serait-elle qu'un feu de paille lié aux vains espoirs des années 1920 ?

La seconde question tient à la dissymétrie de l'approche. Laura Pettinaroli a consulté un certain nombre de fonds d'archives russes, redevenues moins accessibles aujourd'hui, mais le gros de son travail repose sur le dépouillement des archives vaticanes et sur l'utilisation des travaux qui en ont été tirés. La relation qu'elle reconstitue de façon magistrale est donc vue de Rome. On ne pouvait lui demander plus. Mais on se prend à rêver d'un travail complémentaire qui restaurerait la partie russe de la relation. Et qui répondrait à la question qu'on ne peut manquer de se poser : y a-t-il une "politique catholique » de la Russie, tsariste puis soviétique, et dans l'affirmative, quelle est son importance, comparée à la "politique russe » du Saint-Siège ? La réponse devrait prendre en compte, non seulement les forces et pouvoirs politiques, mais aussi les forces religieuses. Laura Pettinaroli note de façon fugace le scandale suscité dans les milieux orthodoxes de l'émigration par les tentatives de négociation du Saint-Siège avec les Soviets. Nul doute que le saint-synode et le patriarcat de Moscou ont aussi leur opinion sur une "politique russe » ayant pour but de convertir la Russie au catholicisme et de la faire revenir dans le giron romain, alors qu'elle est chrétienne depuis près d'un millénaire et qu'elle est convaincue d'avoir conservé la vraie foi, faussée par Rome ! Le refus de la latinisation et tout autant de l'union dans le rite oriental, considéré comme une duperie, sont une constante identitaire de l'Église russe. Les dirigeants de Petrograd ou de Moscou, quels qu'ils soient, ont conscience pour leur part d'être à la tête d'une grande puissance dont les interlocuteurs ou adversaires naturels sont les autres grandes puissances, pas les

6. Voir le travail comparable, par son poids et sa qualité, d'Olivier Sibre, Le Saint-Siège et l'Extrême-Orient, Chine, Corée, Japon de Léon XIII à Pie XII, 1880-1952, Rome, Collection de l'École française de Rome, 459, 2012. 
Églises. Sans revenir sur l'expression mythique attribuée à Staline, « le Vatican, combien de divisions ? " ${ }^{7}$, on peut penser qu'une telle politique de puissance empêche la Russie d'attribuer à sa relation au Saint-Siège un rôle comparable à celui de sa relation aux autres grands de ce monde. Une reconnaissance de jure par Rome serait certes bonne à prendre, mais pas aussi vitale pour les Soviets que celle de l'Allemagne ou des États-Unis. D'autant que, vue de Moscou, la relation n'est pas seulement affaire de diplomatie, mais aussi de politique et d'idéologie. Comment le premier État fondé sur le matérialisme marxiste pourrait-il se compromettre avec un des principaux vecteurs de l'opium du peuple? Sans avoir le loisir d'approfondir le sujet, Laura Pettinaroli note les divergences sur ce point entre les diplomates de la mouvance de Tchitchérine, favorables à la négociation, et les politiques ou les idéologues, bien plus réticents parce que plus soucieux d'orthodoxie doctrinale antireligieuse. Or les premiers sombreront dans les purges staliniennes. Il n'est donc pas sûr qu'il y ait une "politique catholique " de la Russie soviétique. Ou alors il y en a deux, avec une oscillation entre conciliation et répression.

Difficile pourtant de nier, après avoir lu Sophie Coeuré et Laura Pettinaroli, que la révolution bolchevique de 1917 ait suscité au sein du catholicisme et jusqu'en son sommet des vocations russes qui y ont vu à juste titre un défi majeur pour la foi chrétienne. Loin de porter sur le nouveau régime une condamnation immédiate et sans appel, Pierre Pascal a fait un long bout de chemin avec lui; fort de l'appui de Pie XI, Michel d'Herbigny a cru pour sa part à la possibilité de planter à nouveau l'Église catholique en Russie soviétique. En 1933, le premier regagne la France et le second doit quitter Rome. Leur éloignement de la Russie, sinon des choses russes, est certes un aveu d'échec. Il n'en reste pas moins que tous les deux, et bien d'autres du haut en bas de la pyramide ecclésiale, ont cru que la chute du tsarisme était une chance pour le catholicisme en Russie, chance à saisir quel que soit le prix à payer. C'est la mise en valeur de ce moment russe de la pensée et de l'action catholiques qui fait le prix des deux ouvrages recensés.

Étienne FOUILLOUX

Laboratoire de recherche historique Rhône-Alpes, LARHRA, UMR 5190 etienne.fouilloux@wanadoo.fr

7. Voir le récent essai de Frédéric Le Moal, Les divisions du pape. Le Vatican face aux dictatures, Paris, Perrin, 2016 ; après la synthèse de Philippe Chenaux, L'Église catholique et le communisme en Europe (1917-1989). De Lénine à Jean-Paul II, Paris, Le Cerf, 2009. 
